\section{Revista Española de \\ Cirugía Oral y \\ Maxilofacial}

Caso clínico

\title{
Distracción osteogénica vertical de injerto microvascularizado de peroné para reconstrucción mandibular y optimización de la rehabilitación implantológica
}

\author{
G.Y. Cho Lee ${ }^{a, *}$, L. Naval Gías ${ }^{b}$, F.J. Rodríguez Campo ${ }^{b}$, M.F. Muñoz Guerra ${ }^{b}$, \\ J. Sastre Pérez ${ }^{b}$, R. González García ${ }^{c}$, P.L. Martos Díaz ${ }^{d}$ y F.J. Díaz González ${ }^{e}$ \\ a Médico Residente, Servicio de Cirugía Oral y Maxilofacial, Hospital Universitario de La Princesa, Madrid, España. \\ b Médico Adjunto, Servicio de Cirugía Oral y Maxilofacial, Hospital Universitario de La Princesa, Madrid, España. \\ cMédico Adjunto, Servicio de Cirugía Oral y Maxilofacial, Hospital Universitario Infanta Cristina, Badajoz, España. \\ d Médico Adjunto, Servicio de Cirugía Oral y Maxilofacial, Hospital Universitario Nuestra Señora de Candelaria, Santa Cruz de Tenerife, \\ España. \\ eJefe de Servicio, Servicio de Cirugía Oral y Maxilofacial, Hospital Universitario de La Princesa, Madrid, España.
}

\section{INFORMACIÓN DEL ARTÍCULO}

Historia del artículo:

Recibido el 5 de octubre de 2009

Aceptado el 12 de diciembre de 2009

Palabras Clave:

Distracción osteogénica vertical;

Injerto de peroné;

Rehabilitación implantológica

\section{R E S U M E N}

Introducción: En la actualidad, se considera que el injerto microvascularizado de peroné es la mejor opción de tratamiento para las reconstrucciones mandibulares de defectos óseos mayores de $6 \mathrm{~cm}$. No obstante, debido a su limitada altura, presenta una importante desventaja en cuanto a la rehabilitación protésica, particularmente en aquellos casos de resecciones mandibulares parciales con una hemimandíbula contralateral dentada.

Material y métodos: Presentamos un caso clínico de mixoma mandibular tratado mediante resección quirúrgica y reconstrucción mandibular primaria mediante injerto microvascularizado de peroné, al que se sometió a distracción osteogénica vertical para optimizar la posterior rehabilitación implantológica.

Resultados: La discrepancia de altura se solucionó completamente y se colocaron tres implantes osteointegrados en el área distraída, lo cual permitió una rehabilitación protésica muy satisfactoria, tanto estética como funcionalmente.

Conclusiones: La distracción osteogénica vertical de peroné constituye una excelente opción terapéutica para igualar la altura del peroné a la del reborde alveolar de la hemimandíbula dentada contralateral, tras la reconstrucción de defectos mandibulares extensos, de cara a una posterior rehabilitación implantológica, lo que permite llevar a cabo ésta con resultados, tanto funcionales como estéticos, muy satisfactorios. Además, no presenta los inconvenientes de otras técnicas empleadas en estos pacientes, como el peroné en doble barra (mayor dificultad técnica y mayor riesgo de trombosis en el pedículo) y el empleo de injertos "onlay" (morbilidad añadida en la zona de toma del injerto).

(c) 2009 SECOM. Publicado por Elsevier España, S.L. Todos los derechos reservados.

\footnotetext{
*Autor para correspondencia.

Correo electrónico: gui-youncho@hotmail.com; choguiyoun@gmail.com (G.Y. Cho Lee).
} 
Keywords:

Vertical distraction osteogenesis; Fibula flap;

Prosthetic rehabilitation

\section{Vertical distraction osteogenesis of microvascularised fibular grafts for mandibular reconstruction and optimisation of rehabilitation implantology}

\author{
A B S T R A C T
}

Introduction: Free vascularized fibular flap is considered the treatment of choice in mandibular reconstruction for extensive bone defects. But, when the reconstruction involves a dentate mandible, the fibula has the limit as it doesn't offer sufficient bone height to restore the alveolar arch up to the occlusal plane. Therefore, the deficiency in bone height makes implant placement impractical.

Materials and methods: We report a case of vertical distraction osteogenesis of a free vascularized fibula flap used to reconstruct a hemimandible after resection of an odontogenic myxoma. The distraction device was applied intraorally. Distraction of $0.5 \mathrm{~mm}$ per day was performed followed by 3 months of consolidation period.

Results: The vertical discrepancy between the fibula and the native hemimandible was corrected. The increase of vertical bone height was stable and enabled placement of dental implants without any complications.

Conclusions: We believe that vertical distraction osteogenesis of free vascularized flaps is a reliable technique that optimizes implant positioning for ideal prosthetic rehabilitation, after mandibular reconstruction following tumour surgery.

() 2009 SECOM. Published by Elsevier España, S.L. All rights reserved.

\section{Introducción}

En la actualidad, se considera que el injerto microvascularizado de peroné es la mejor opción de tratamiento para las reconstrucciones mandibulares de defectos óseos mayores de $6 \mathrm{~cm}$ que resultan de traumatismos, infecciones o resecciones tumorales ${ }^{1}$. Desde 1989, cuando Hidalgo empleó por primera vez el injerto microvascularizado de peroné como nuevo método de reconstrucción mandibular, éste se ha convertido en un procedimiento eficaz, seguro y predecible, que aporta resultados excelentes tanto estéticos como funcionales ${ }^{2}$.

El peroné presenta numerosas características anatómicas que resultan beneficiosas para su empleo como método reconstructivo: longitud ósea adecuada (disponibilidad de más de $20 \mathrm{~cm}$ de hueso), aporte vascular perióstico y medular doble (lo cual permite la realización de múltiples osteotomías), adecuada longitud del pedículo vascular, baja morbilidad del sitio donante, geometría constante, y calidad (estructura bicortical) y volumen óseos idóneos para la colocación de implantes osteointegrados ${ }^{3-5}$.

No obstante, el peroné, debido a su limitada altura (15 mm como máximo), presenta una importante desventaja en cuanto a la rehabilitación protésica, particularmente en aquellos casos de resecciones mandibulares parciales con una hemimandíbula contralateral dentada. La discrepancia de altura entre el injerto de peroné y el reborde alveolar de la hemimandíbula dentada contralateral supone una dificultad importante para que ésta se lleve a cabo satisfactoriamente. Esta discrepancia de altura conduciría a una sobrecarga de fuerzas sobre los implantes, lo cual afectaría a la supervivencia de los mismos a largo plazo ${ }^{6-12}$.

Para solucionar este problema se han empleado técnicas como la aposición de injertos "onlay" y la técnica del injerto de peroné en doble barra. Sin embargo, éstas han obtenido resultados limitados y presentan grandes dificultades técni- cas $^{13}$. Por ello, se ha ideado la distracción osteogénica vertical del injerto microvascularizado de peroné6-12.

La distracción osteogénica se emplea rutinariamente para aumentar la altura ósea de mandíbulas edéntulas con reabsorción ósea vertical (distracción alveolar). Con ello se consigue aumentar el volumen óseo para la colocación de implantes osteointegrados. Sin embargo, son muy pocos los casos en los que se ha empleado la distracción osteogénica en injertos microvascularizados de peroné para aumentar su altura ${ }^{6}$.

Presentamos un caso clínico de mixoma mandibular tratado mediante resección quirúrgica y reconstrucción mandibular primaria mediante injerto microvascularizado de peroné, al que se sometió a distracción osteogénica vertical para optimizar la posterior rehabilitación implantológica. La discrepancia de altura se solucionó completamente y se colocaron tres implantes osteointegrados en el área distraída, lo cual permitió una rehabilitación protésica muy satisfactoria, tanto estética como funcionalmente.

\section{Caso clínico}

Paciente varón de 33 años, que fue remitido desde otro centro hospitalario por presentar tumoración hemimandibular derecha de gran tamaño, de 6 meses de evolución, que había experimentado un crecimiento progresivo, y que refería tras la realización de exodoncia de molar del cuarto cuadrante, sin alusión a padecimiento de dolor importante a dicho nivel.

La exploración física (fig. 1) y la ortopantomografía (fig. 2) evidenciaron una tumoración dependiente de hueso de 5 $6 \mathrm{~cm}$ de diámetro, con afectación de ambas corticales óseas, que se extendía desde la región del segundo premolar hasta la mitad de la rama mandibular derecha, con afectación del músculo pterigoideo interno ipsilateral. 


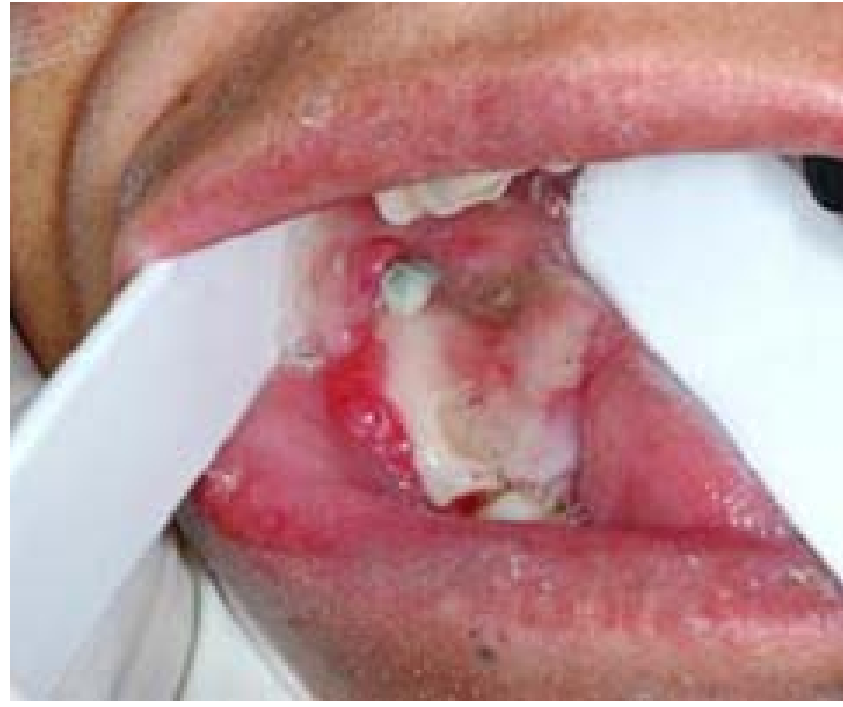

Figura 1 - Tumoración de gran tamaño en hemimandíbula derecha. Se puede observar el modelado de la masa tumoral por mordida de la hemiarcada superior.

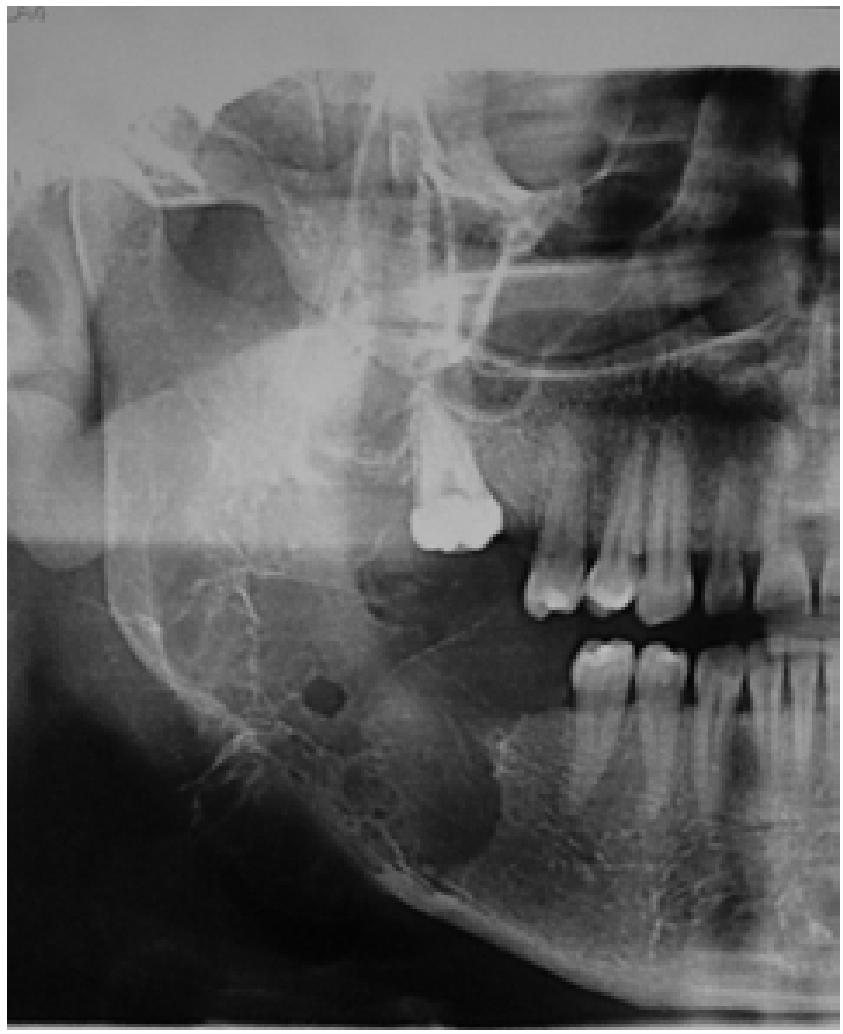

Figura 2 - Ortopantomografía: gran lesión radiolúcida que destruye la lámina basal y cortical mandibular.

Se practicó una tomografía computarizada (TC) facial (fig. 3) que demostró una lesión tumoral mandibular de 5,5 $\times$ $4,8 \times 3 \mathrm{~cm}$, que destruía la cortical mandibular interna desplazando la musculatura y las estructuras de la base de la lengua, y que afectaba la mayor parte del cuerpo y rama man-

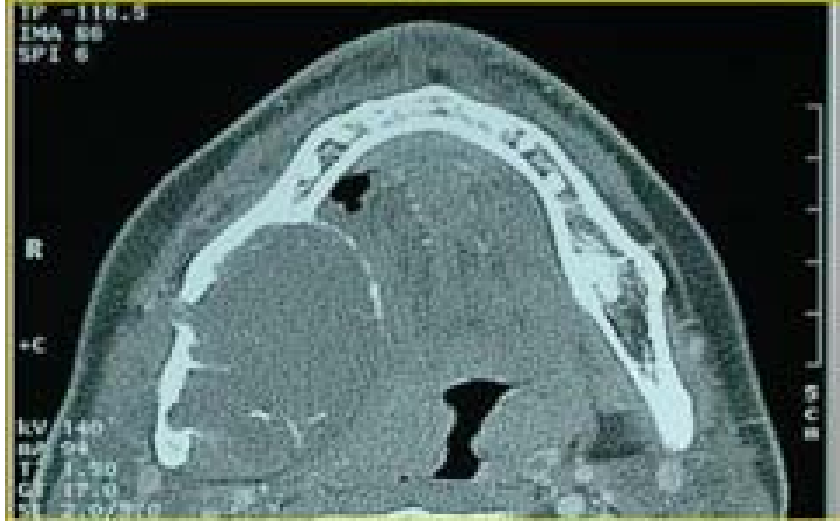

Figura 3 - Tomografía computarizada: masa en hemimandíbula derecha que destruye ambas corticales óseas.

dibulares derechos, con preservación del cóndilo. Se realizó biopsia diagnóstica, con el resultado de lesión mixoide con componente inflamatorio linfoplasmocitario reactivo, con positividad para vimentina, CD138, Kappa, Lambda y con positividad parcial para actina. La inmunohistoquímica mostró negatividad para S-100, CD31 y CD68. El diagnóstico fue de mixoma odontogénico mandibular.

Bajo anestesia general e intubación nasotraqueal, se realizó extirpación quirúrgica de la lesión con márgenes, mediante abordaje combinado intra y extraoral, practicándose hemimandibulectomía segmentaria desde la región canina hasta la rama mandibular y apófisis coronoides ipsilaterales, preservándose el cóndilo mandibular. El paciente presentó una hemimandíbula contralateral dentada. Se realizó reconstrucción primaria mediante injerto libre microvascularizado de peroné. El espesor máximo del peroné fue de $14 \mathrm{~mm}$. El colgajo fue preformado in situ, con el tallado de la nueva hemimandíbula mediante una barra de reconstrucción modelada sobre la mandíbula original, y la posterior realización de la osteotomía del hueso peroneal y su fijación con miniplacas. Se realizó anastomosis vascular entre las arterias peronea y facial derechas, y entre las venas concomitante peronea y tronco venoso tirolinguofacial derechos. El estudio histológico de la pieza fue informado como mixoma odontogénico mandibular sin afectación de bordes quirúrgicos. El postoperatorio transcurrió sin complicaciones. Tras un año de la intervención, al observar una discrepancia de altura entre el peroné y el reborde alveolar de la hemimandíbula contralateral de $17 \mathrm{~mm}$ (fig. 4), se decidió realizar distracción osteogénica vertical del peroné mediante colocación de un distractor alveolar intraoral.

La colocación del distractor se realizó bajo anestesia general. El injerto de peroné fue expuesto mediante una incisión en la mucosa oral vestibular, tras lo cual se procedió a realizar una disección subperióstica para visualizar adecuadamente el hueso subyacente, poniendo extremado cuidado en preservar el periostio de la cara lingual del peroné.

El distractor alveolar intraoral (MODUS ARS 1.5; Medartis ${ }^{\circledR}$, Basel, Suiza) se colocó sobre la superficie vestibular del peroné para diseñar correctamente las osteotomías. Las osteotomías se realizaron sobre la cara vestibular del peroné, mediante el uso de una sierra oscilante, con irrigación con 


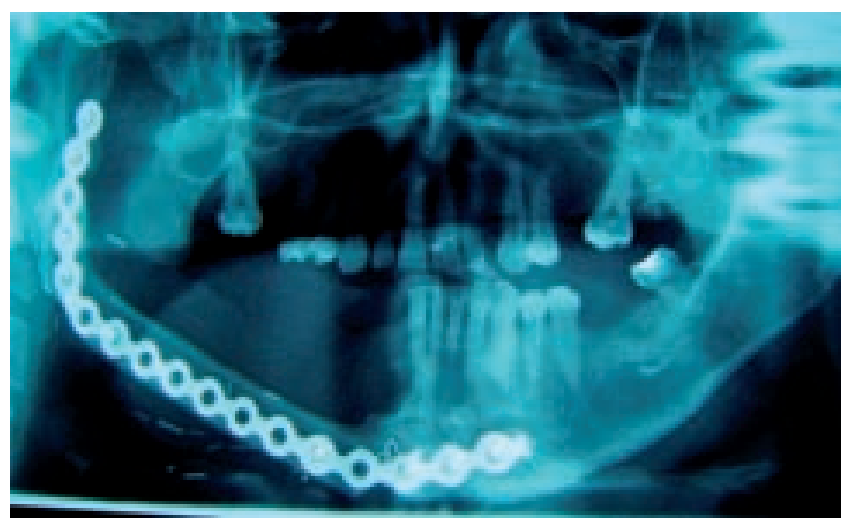

Figura 4 - Ortopantomografía tras la reconstrucción mandibular con injerto microvascularizado de peroné. Obsérvese la discrepancia de altura entre el injerto de peroné y la hemimandíbula contralateral dentada.

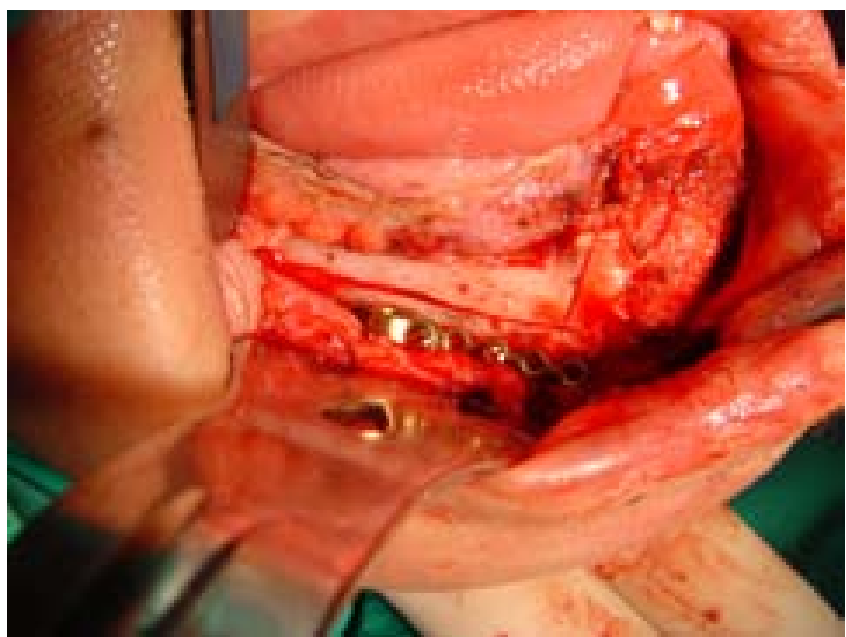

Figura 5 - Imagen intraoperatoria. Osteotomías horizontal y vertical del peroné.

suero salino. Se obtuvo así un segmento óseo trapezoidal, habiendo mantenido íntegro el periostio lingual (fig. 5). Se fijó el distractor alveolar mediante tornillos, a ambos lados de la osteotomía horizontal (fig. 6). Una vez comprobado el perfecto funcionamiento del distractor, se dispuso el mismo en su posición de inicio, de tal manera que ambos fragmentos de hueso estuvieran en perfecta aposición. Esto es fundamental para iniciar la formación de hueso desde la posición de máximo contacto entre fragmentos y para lograr una adecuada hemostasia postosteotomía. Se procedió al cierre de la incisión vestibular dejando parte del distractor a través de la incisión, con el fin de facilitar la activación del mismo.

Durante el periodo de latencia (10 días) no se realizó la activación del distractor. Después de esto, se procedió a la misma, a razón de 0,5 mm diarios. En los controles ortopantomográficos seriados (obtenidos semanalmente) se observó la distracción vertical del hueso peroneo y la formación de trabéculas óseas en la interfase al final de la estabilización

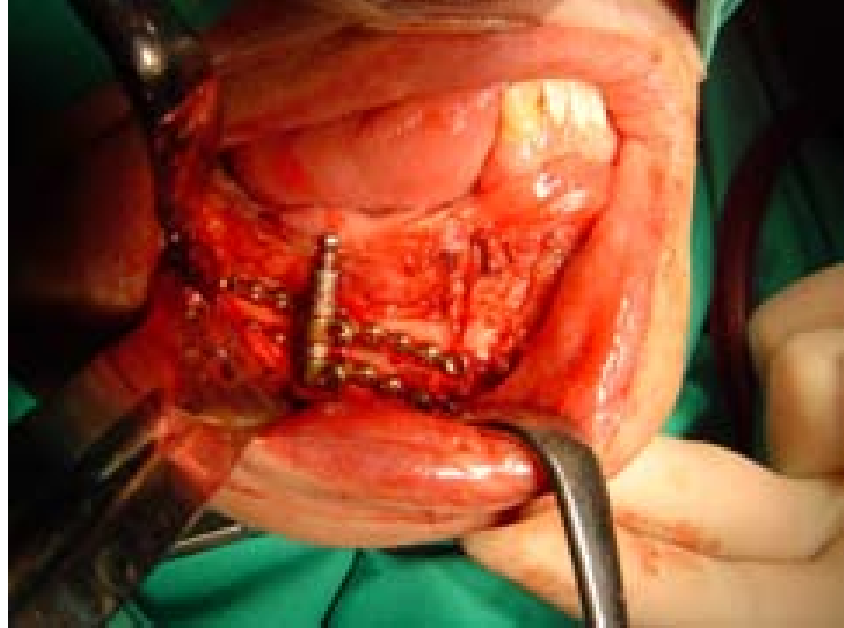

Figura 6 - Distractor alveolar intraoral colocado y fijado al peroné mediante tornillos, una vez realizadas las osteotomías.

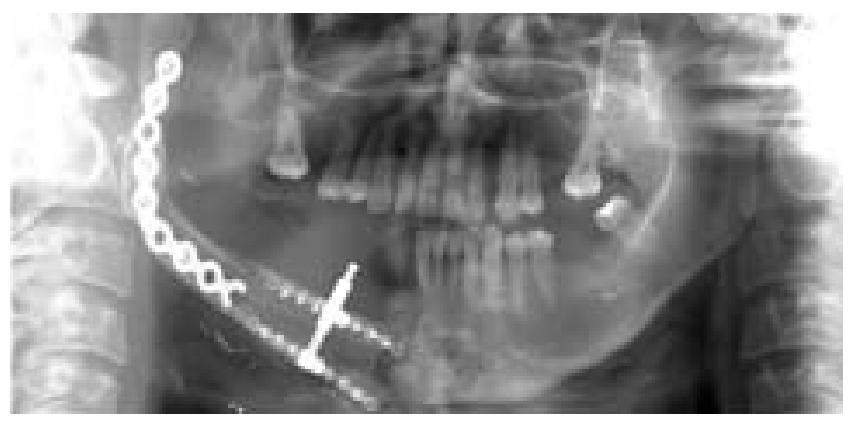

Figura 7 - Ortopantomografía. En la mitad de la distracción. Se observa la distracción vertical del hueso peroneo y la formación de trabéculas óseas en la interfase.

(figs. 7 y 8). Se obtuvieron $17 \mathrm{~mm}$ de hueso en 34 días, de manera que el injerto de peroné alcanzó el mismo nivel que el reborde alveolar contralateral, con una corrección óptima de la discrepancia de altura inicial entre ambos.

El periodo de consolidación duró tres meses, durante los cuales el distractor fue mantenido en su lugar de fijación inicial. La ortopantomografía a los tres meses confirmó una excelente osificación entre la basilar y el fragmento distraído (fig. 8). Posteriormente, en una segunda intervención, se procedió a la retirada del distractor mediante el mismo abordaje intraoral. La calidad del hueso neoformado, comprobado de manera macroscópica, fue excelente (fig. 9). En la misma intervención se colocaron tres implantes roscados de titanio (Mozo-Grau ${ }^{\circledR}$ ) de 3,75 × $15 \mathrm{~mm}$, en el área distraída. Se consiguió una buena estabilidad primaria en todos los implantes (figs. 10 y 11). El postoperatorio transcurrió sin complicaciones. La rehabilitación protésica, mediante una prótesis implantosoportada, se llevó a cabo con éxito tras tres meses de periodo de osteointegración (figs. 12 y 13). Los resultados obtenidos, tanto estéticos como funcionales, fueron satisfactorios tanto para el paciente como para el cirujano, tras 18 meses de seguimiento. 


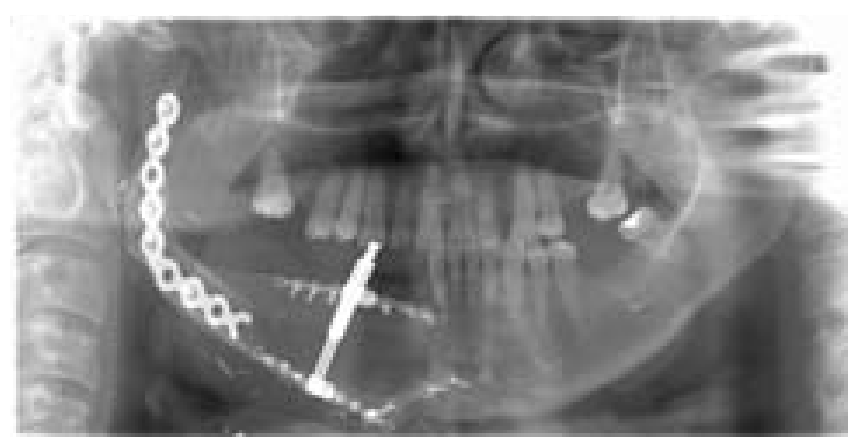

Figura 8 - Al final del periodo de consolidación (tres meses). La ortopantomografía confirma una excelente osificación entre la basilar y el fragmento distraído.

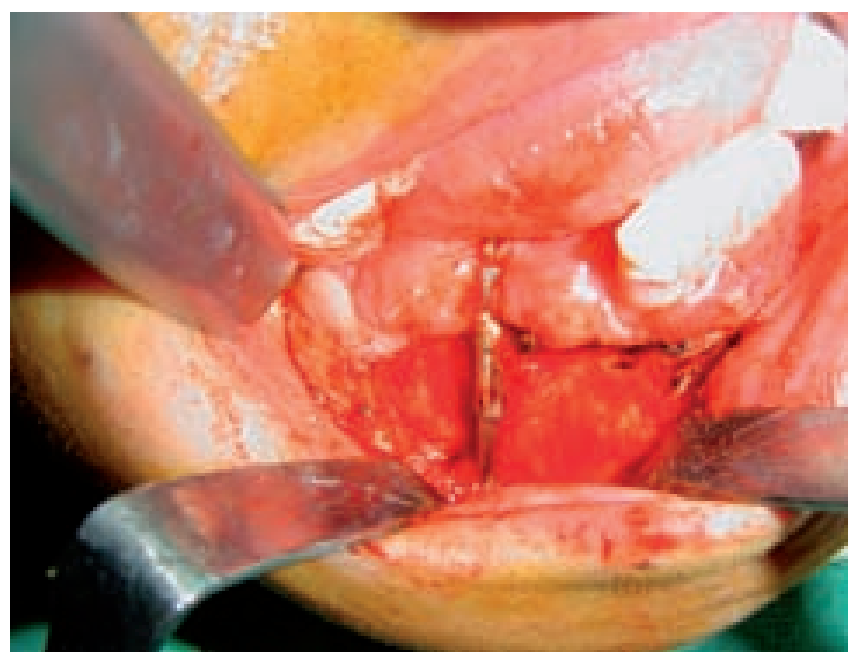

Figura 9 - Imagen intraoperatoria. La calidad del hueso neoformado, comprobado de manera macroscópica, es excelente. Se obtuvieron $17 \mathrm{~mm}$ de hueso, de manera que el injerto de peroné alcanzó el mismo nivel que el reborde alveolar contralateral, con una corrección óptima de la discrepancia de altura inicial entre ambos.

\section{Discusión}

Se han descrito numerosas técnicas a lo largo de los años para la reconstrucción de defectos mandibulares que resultan de traumatismos, infecciones o resecciones quirúrgicas. En la actualidad, se considera que el empleo de injertos microvascularizados y de técnicas microquirúrgicas es seguro, eficaz y predecible ${ }^{6}$.

El injerto microvascularizado de peroné presenta muchas ventajas para la reconstrucción mandibular, en comparación con otros injertos microvascularizados, que lo convierten en la mejor opción para la reconstrucción mandibular. No obstante, su principal limitación es la altura insuficiente, que impide restaurar la basilar mandibular y el reborde alveolar simultáneamente, en el lado afecto. Este aspecto resulta especialmente relevante en los casos de resecciones parciales de mandíbulas dentadas. Este defecto de altura ósea del peroné impide que la rehabilitación implantológica sea satisfactoria, tanto funcional como estéticamente. La discrepancia de altura entre el peroné y el reborde alveolar del lado sano no permite una correcta rehabilitación con una prótesis implantomucosoportada o implantosoportada ${ }^{6-12}$.

Desde el punto de vista funcional, los implantes necesitarían sostener prótesis con coronas muy largas para compensar la distancia intermaxilar y llegar al plano oclusal, con el consiguiente sometimiento de la prótesis a fuerzas desfavorables y de sobrecarga de los implantes. Todo ello afectaría negativamente a la supervivencia a largo plazo de los implantes ${ }^{6,7}$. En un estudio reciente se comprobó que, por cada milímetro de aumento de la altura de la corona, el incremento de las fuerzas de carga sería de un $20 \%{ }^{13}$. Desde el punto de vista estético, el empleo de prótesis con coronas muy largas representa un problema notable ${ }^{6,7}$.

Para solventar estas dificultades, el injerto de peroné se podría colocar al mismo nivel que el reborde alveolar de la hemimandíbula contralateral, pero esto afectaría negativamente al contorno facial resultante ${ }^{6-12}$. Otra alternativa sería el empleo de la técnica del injerto de peroné en doble barra, descrita por Horiuchi et al (1995) y Bähr et al (1998) ${ }^{13}$. Esta técnica permite duplicar la altura del injerto óseo de peroné. Su principal inconveniente es que, en el caso de defectos grandes, la longitud del peroné puede resultar insuficiente para duplicar la altura y cubrir completamente el defecto, simultáneamente. Además de ser un método técnicamente complejo, presenta un mayor riesgo de trombosis del pedículo. Una tercera posibilidad es el empleo de otro injerto microvascularizado o la aposición "onlay" de otro injerto óseo, sobre el peroné. Esta alternativa no se suele realizar por conllevar una alta morbilidad añadida en un paciente que ha sido sometido a una intervención quirúrgica de gran envergadura ${ }^{6-12}$.

Para evitar todos estos riesgos, se ha ideado la distracción osteogénica vertical de peroné. La aplicación de los principios de la distracción osteogénica representa una solución a este problema ${ }^{6}$. Este método se ha empleado para la distracción alveolar intraoral en casos de reabsorción ósea vertical en mandíbulas edéntulas, para incrementar el volumen óseo y permitir la colocación de implantes osteointegrados. Según el conocimiento de los autores, este método se ha empleado en muy pocos casos sobre injertos de peroné6-12.

La distracción osteogénica vertical de peroné no precisa de la realización de una segunda intervención mayor y, por lo tanto, no añade más morbilidad. Además, aporta una ventaja fundamental al distraer también los tejidos blandos y adaptarlos a la nueva altura ósea sin riesgo de crear tensión tisular ni de exposición del injerto. A su vez, la discrepancia de altura puede corregirse de acuerdo a las necesidades de cada caso, mediante un método relativamente sencillo e inocuo ${ }^{6-12}$.

Los parámetros óptimos (periodo de latencia, ritmo de distracción y periodo de consolidación) son desconocidos para este tipo de distracción ${ }^{9,12}$. Por ello, estos parámetros fueron extrapolados de nuestra experiencia en casos de distracción mandibular y de los estudios de distracción osteogénica craneofacial ${ }^{14,15}$. Así, el periodo de latencia fue de 10 días, el ritmo de distracción fue de $0,5 \mathrm{~mm}$ por día y el periodo de consolidación fue de tres meses. Asimismo, se tuvieron en cuenta algunos recursos técnicos importantes: a) es impor- 


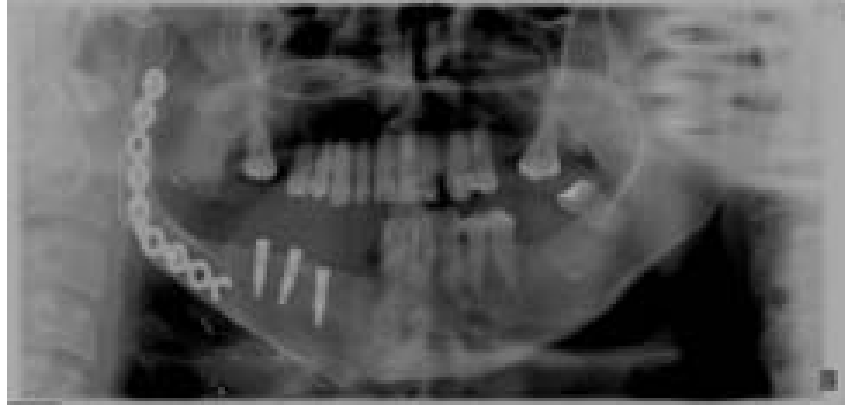

Figura 10 - Ortopantomografía. Tres implantes osteointegrados en el área distraída

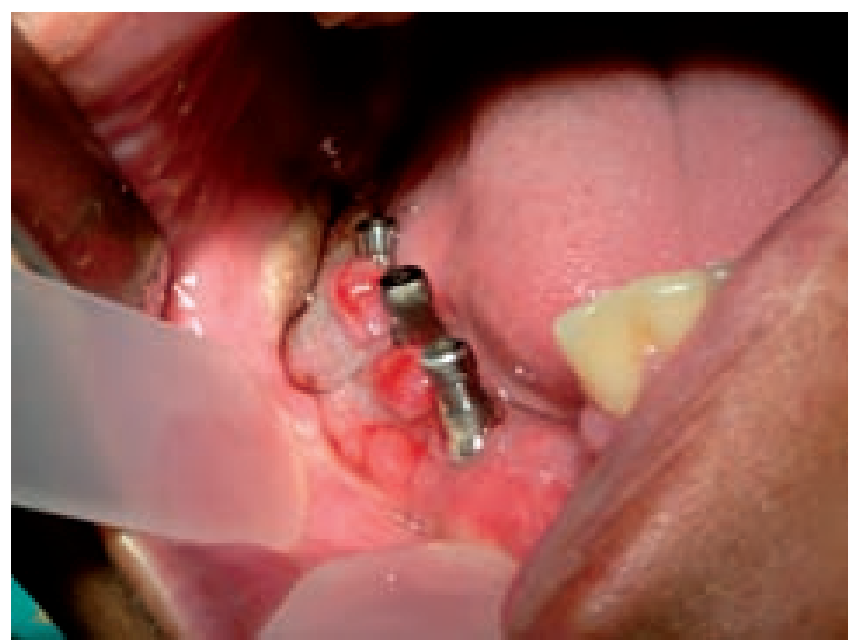

Figura 11 - Aspecto clínico intraoral del paciente una vez colocados los implantes. Altura alveolar adecuada.

tante mantener la integridad del periostio para asegurar un correcto aporte sanguíneo, y b) el vector de distracción fue perpendicular al eje mayor del peroné y, por tanto, a la orientación de las trabéculas óseas.

El crecimiento óseo fue claro y evidente en el área distraída, obteniéndose $17 \mathrm{~mm}$ de hueso neoformado en 34 días. El aporte sanguíneo proporcionado por el periostio, en el caso del peroné, es más importante que el proporcionado por el endostio y, por lo tanto, su integridad es un factor determinante en el éxito del procedimiento ${ }^{12,13}$. Por ello, se esperaron 12 meses desde la primera intervención (resección tumoral y reconstrucción mediante injerto microvascularizado de peroné) hasta la colocación del distractor óseo. Durante el periodo de distracción y el seguimiento del paciente, se realizaron controles radiológicos mediante ortopantomografías seriadas (figs. 7 y 8) que demostraron la correcta formación de hueso en el área distraída y su mantenimiento durante el seguimiento de 18 meses (figs. 10 y 12).

El paciente fue rehabilitado con éxito mediante una prótesis implantosoportada y, tras un año y medio de carga funcional, no se observan signos de reabsorción ósea alrededor de los implantes y la altura ósea se ha mantenido intacta (fig. 12).

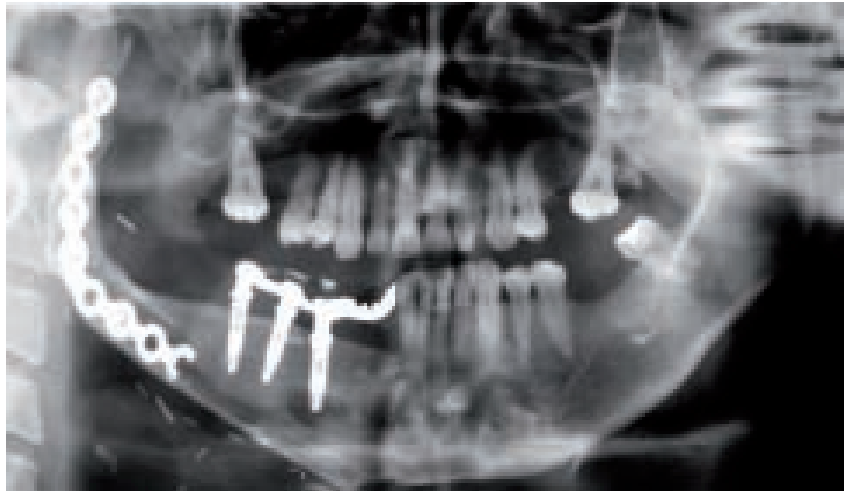

Figura 12 - Ortopantomografía. Prótesis implantosoportada sobre implantes osteointegrados en el área distraída.

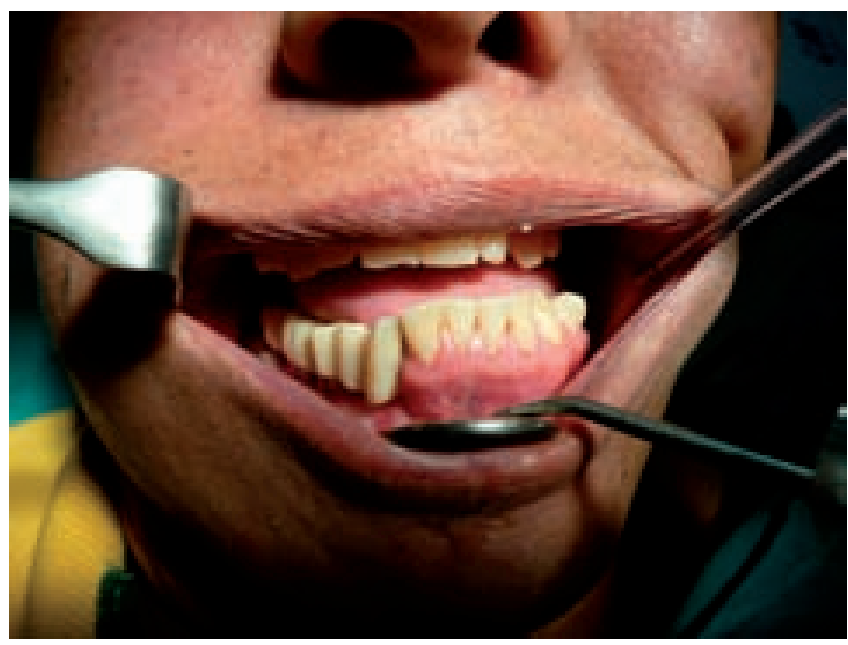

Figura 13 - Aspecto clínico intraoral del paciente finalizado el procedimiento, con la prótesis definitiva implantosoportada.

\section{Conclusiones}

La distracción osteogénica vertical de peroné constituye una excelente opción terapéutica para igualar la altura del peroné a la del reborde alveolar de la hemimandíbula dentada contralateral, tras la reconstrucción de defectos mandibulares extensos, de cara a una posterior rehabilitación implantológica, permitiendo llevar a cabo ésta con resultados, tanto funcionales como estéticos, muy satisfactorios. Además, no presenta los inconvenientes de otras técnicas empleadas en estos pacientes, como el peroné en doble barra (mayor dificultad técnica y mayor riesgo de trombosis en el pedículo) y el empleo de injertos "onlay" (morbilidad añadida en la zona de toma del injerto). El volumen óseo obtenido es estable en el tiempo. En nuestra opinión, esta modalidad de distracción debería estandarizarse y convertirse en el tratamiento de la discrepancia ósea entre el injerto de peroné y la hemimandíbula dentada contralateral, tras resecciones mandibulares parciales. 


\section{B I B L I O G R A F Í A}

1. Cordeiro PG, Disa JJ, Hidalgo DA, Hu QY. Reconstruction of the mandible with osseous free flaps: a 10-year experience with 150 consecutive patients. Plast Reconstr Surg. 1999;104: 1314-20.

2. Hidalgo DA. Free fibula flap: a new method of mandible reconstruction. Plast Reconstr Surg. 1989;84:71-9.

3. Hayter JP, Cawood JI. Oral rehabilitation with endosteal implants and free flaps. Int J Oral Maxillofac Surg. 1996;25:3-12

4. Moscoso JF, Keller J, Genden E, Weinberg H, Biller HF, Buchbinder D, et al. Vascularized bone flaps in oromandibular reconstruction. A comparative anatomic study of bone stock from various donor sites to assess suitability for enosseous dental implants. Arch Otolaryngol Head Neck Surg. 1994;120:36-43.

5. Chang YM, Santamaria E, Wei FC, Chen HC, Chan CP, Shen YF, et al. Primary insertion of osseointegrated dental implants into fibula osteoseptocutaneous free flap for mandible reconstruction. Plast Reconstr Surg. 1998;102:680-8.

6. Chiapasco M, Brusati R, Galioto S. Distraction osteogenesis of a fibular revascularized flap for improvement of oral implant positioning in a tumor patient: a case report. J Oral Maxillofac Surg. 2000;58:1434-40.

7. Kürkü M, Benlidayi ME, Kurtoglu C, Kesiktas E. Placement of implants in the mandible reconstructed with free vascularized fibula flap: comparison of 2 cases. Oral Surg Oral Med Oral Pathol Oral Radiol Endod. 2008;105:e36-e40.

8. Schleier P, Hyckel P, Fried W, Beinemann J, Wurdinger J, Hinz $\mathrm{M}$, et al. Vertical distraction of fibula transplant in a case of mandibular defect caused by shotgun injury. Int J Oral Maxillofac Surg. 2006;35:861-4.

9. Eski M, Turegun M, Deveci M, Gokce HS, Sengezer M. Vertical distraction osteogenesis of fibular bone flap in reconstructed mandible. Ann Plast Surg. 2006;57:631-6.

10. Ortakoglu K, Suer BT, Ozyigit A, Ozen T, Sencimen M. Vertical distraction osteogenesis of fibula transplant for mandibular reconstruction: a case report. Oral Surg Oral Med Oral Pathol Oral Radiol Endod. 2006;102:e8-e11.

11. Klesper B, Lazar F, Siessegger M, Hidding J, Zöller JE. Vertical distraction osteogenesis of fibula transplants for mandibular reconstruction-a preliminar study. J Craniomaxillofac Surg. 2002;30:280-5.

12. Nocini PF, Wangerin K, Albanese M, Kretschmer W, Cortelazzi $R$. Vertical distraction of a free vascularized fibula flap in a reconstructed hemimandible: case report. J Craniomaxillofac Surg. 2000;28:20-4.

13. Bähr W, Stoll P, Wachter R. Use of "double-barrel" free vascularized fibula in mandibular reconstruction. J Oral Maxillofac Surg. 1998;56:38-44.

14. González-García R, Rubio-Bueno P, Naval-Gías L, RodríguezCampo FJ, Escorial-Hernández V, Martos PL, et al. Internal distraction osteogenesis in mandibular reconstruction: clinical experience in 10 cases. Plast Reconstr Surg. 2008;121:563-75.

15. Rubio-Bueno P, Naval-Gías L, Rodríguez-Campo FJ, Gil-Díez JL, Díaz-González FJ. Internal distraction osteogenesis for reconstruction of mandibular segmental defects: preliminary clinical experience in five cases. J Oral Maxillofac Surg. 2005;63:598-608. 